\title{
First results from the CRESST-III low-mass dark matter program
}

A. H. Abdelhameed, ${ }^{1}$ G. Angloher, ${ }^{1}$ P. Bauer, ${ }^{1, *}$ A. Bento, ${ }^{1,8}$ E. Bertoldo, ${ }^{1}$ C. Bucci, ${ }^{2}$ L. Canonica, ${ }^{1}$ A. D’ Addabbo, ${ }^{2,9}$ X. Defay, ${ }^{3}$ S. Di Lorenzo, ${ }^{2,9}$ A. Erb, ${ }^{3,10}$ F. v. Feilitzsch, ${ }^{3}$ S. Fichtinger, ${ }^{4}$ N. Ferreiro Iachellini, ${ }^{1}$ A. Fuss, ${ }^{4,5}$ P. Gorla, ${ }^{2}$ D. Hauff, ${ }^{1}$ J. Jochum, ${ }^{6}$ A. Kinast, ${ }^{3}$ H. Kluck, ${ }^{4,5}$ H. Kraus, ${ }^{7}$ A. Langenkämper, ${ }^{3}$ M. Mancuso, ${ }^{1}$ V. Mokina, ${ }^{4}$ E. Mondragon, ${ }^{3}$ A. Münster, ${ }^{3}$ M. Olmi, ${ }^{2,9}$ T. Ortmann, ${ }^{3}$ C. Pagliarone, ${ }^{2,11}$ L. Pattavina,,${ }^{3,9}$ F. Petricca, ${ }^{1}$ W. Potzel, ${ }^{3}$ F. Pröbst, ${ }^{1}$ F. Reindl@, ${ }^{4,5, \dagger}$ J. Rothe, ${ }^{1}$ K. Schäffner, ${ }^{2,9}$ J. Schieck, ${ }^{4,5}$ V. Schipperges, ${ }^{6}$ D. Schmiedmayer, ${ }^{4,5}$ S. Schönert, ${ }^{3}$ C. Schwertner, ${ }^{4,5}$

M. Stahlberg $\odot,{ }^{4,5,}$ L. Stodolsky, ${ }^{1}$ C. Strandhagen, ${ }^{6}$ R. Strauss, ${ }^{3}$ C. Türkoğlu, ${ }^{4,5}$ I. Usherov, ${ }^{6}$ M. Willers, ${ }^{3}$ and V. Zema ${ }^{2,9,12}$

\section{(CRESST Collaboration)}

\author{
${ }^{1}$ Max-Planck-Institut für Physik, 80805 München, Germany \\ ${ }^{2}$ INFN, Laboratori Nazionali del Gran Sasso, 67010 Assergi, Italy \\ ${ }^{3}$ Physik-Department and Excellence Cluster Universe, \\ Technische Universität München, 85747 Garching, Germany \\ ${ }^{4}$ Institut für Hochenergiephysik der Österreichischen Akademie der Wissenschaften, 1050 Wien, Austria \\ ${ }^{5}$ Atominstitut, Technische Universität Wien, 1020 Wien, Austria \\ ${ }^{6}$ Eberhard-Karls-Universität Tübingen, 72076 Tübingen, Germany \\ ${ }^{7}$ Department of Physics, University of Oxford, Oxford OX1 3RH, United Kingdom \\ ${ }^{8}$ LIBPhys, Departamento de Fisica, Universidade de Coimbra, P3004 516 Coimbra, Portugal \\ ${ }^{9}$ Gran Sasso Science Institute, 67100, L'Aquila, Italy \\ ${ }^{10}$ Walther-Meißner-Institut für Tieftemperaturforschung, 85748 Garching, Germany \\ ${ }^{11}$ Dipartimento di Ingegneria Civile e Meccanica, Universitá degli Studi di Cassino e del Lazio \\ Meridionale, 03043 Cassino, Italy \\ ${ }^{12}$ Chalmers University of Technology, Department of Physics, 41296 Göteborg, Sweden
}

(Received 12 April 2019; published 25 November 2019)

\begin{abstract}
The CRESST experiment is a direct dark matter search which aims to measure interactions of potential dark matter particles in an Earth-bound detector. With the current stage, CRESST-III, we focus on a low energy threshold for increased sensitivity towards light dark matter particles. In this paper we describe the analysis of one detector operated in the first run of CRESST-III (05/2016-02/2018) achieving a nuclear recoil threshold of $30.1 \mathrm{eV}$. This result was obtained with a $23.6 \mathrm{~g} \mathrm{CaWO}_{4}$ crystal operated as a cryogenic scintillating calorimeter in the CRESST setup at the Laboratori Nazionali del Gran Sasso (LNGS). Both the primary phonon (heat) signal and the simultaneously emitted scintillation light, which is absorbed in a separate silicon-on-sapphire light absorber, are measured with highly sensitive transition edge sensors operated at $\sim 15 \mathrm{mK}$. The unique combination of these sensors with the light element oxygen present in our target yields sensitivity to dark matter particle masses as low as $160 \mathrm{MeV} / \mathrm{c}^{2}$.
\end{abstract}

DOI: $10.1103 /$ PhysRevD.100.102002

\section{INTRODUCTION}

Today, the Standard Model of particle physics provides a widely consistent description of the visible matter in the Universe. However, the ever-growing precision of cosmological observations substantiates the finding that the visible matter contributes comparatively little to the matter density of the Universe which is, instead, dominated by dark matter. Numerous experiments strive to decipher

\footnotetext{
*philipp.bauer@mpp.mpg.de

florian.reindl@oeaw.ac.at

*martin.stahlberg@oeaw.ac.at
}

the nature of dark matter, either by a potential production of dark matter particles in collisions of Standard Model particles, by searching for secondary Standard Model particles originating from the annihilation of dark matter particles, or by aiming at the direct observation of interactions of dark matter particles in Earth-bound detectors. As of today, none of these three channels has delivered an unambiguous hint for dark matter particles [1].

Since, in particular, the mass of the dark matter particle(s) is a priori unknown, direct searches for dark matter need to cover the widest possible mass range. This necessarily implies the use of different experimental techniques. In the standard scenario, assuming spin-independent 
and elastic scattering of dark matter particles off nuclei, liquid noble gas experiments take the lead in the high mass range. Solid-state or gas detectors are best suited for light $\left(\lesssim 1 \mathrm{GeV} / \mathrm{c}^{2}\right)$ dark matter due to their lower energy thresholds. For spin-dependent interactions superheated bubble chambers play an important role [2]. In the last years, and also in this article, the sensitivity for dark matter-nucleus scattering was pushed to dark matter particle masses well below $1 \mathrm{GeV} / \mathrm{c}^{2}$, hence approaching mass regimes typically probed with dark matter-electron scattering [3].

The CRESST-III experiment operates scintillating $\mathrm{CaWO}_{4}$ crystals as cryogenic calorimeters, simultaneously measuring a phonon (heat) and a scintillation light signal. A distinctive feature of the phonon signal is a precise determination of the energy deposited in the crystal, independent from the type of particle interaction. This property, in combination with a low energy threshold, makes cryogenic calorimeters particularly suited for lowmass dark matter detection. Contrary to the phonon signal, the scintillation light strongly depends on the type of particle interaction, yielding event-by-event discrimination between the dominant background ( $\beta / \gamma$-interactions) and the sought-for nuclear recoils. Phonon and light signals are acquired by transition edge sensors (TESs) operated at around $15 \mathrm{mK}$ and read out by SQUID amplifiers [4].

In this work we present physics results acquired with detector A, which has the lowest threshold $(30.1 \mathrm{eV})$ among ten detectors of the same design $(\sim 24 \mathrm{~g}$ target mass each) operated in the first run of CRESST-III. In [5] we showed results from a first analysis of detector A; however, this analysis was still based on a hardwaretriggered data subset with an analysis threshold fixed at $100 \mathrm{eV}$.

\section{CRESST-III SETUP AND DATA ACQUISITION}

\section{A. Experimental setup}

The main CRESST infrastructure is located in the Laboratori Nazionali del Gran Sasso (LNGS) underground laboratory in central Italy, which provides an overburden against cosmic radiation with a water equivalent of $3600 \mathrm{~m}$ [6]. Remaining muons are tagged by an active muon veto with $98.7 \%$ geometrical coverage [7]. In addition, the experimental volume is protected by concentric layers of shielding material comprising - from outside to insidepolyethylene, lead, and copper. The polyethylene shields from environmental neutrons, while lead and copper suppress $\gamma$ rays. A second layer of polyethylene inside the copper shielding guards against neutrons produced in the lead or the copper shields.

A commercial ${ }^{3} \mathrm{He} /{ }^{4} \mathrm{He}$-dilution refrigerator provides the base temperature of about $5 \mathrm{mK}$. Cryogenic liquids $\left(\mathrm{LN}_{2}\right.$ and $\left.\mathrm{LHe}\right)$ are refilled three times a week causing a downtime of about $3 \mathrm{~h}$ per refill.

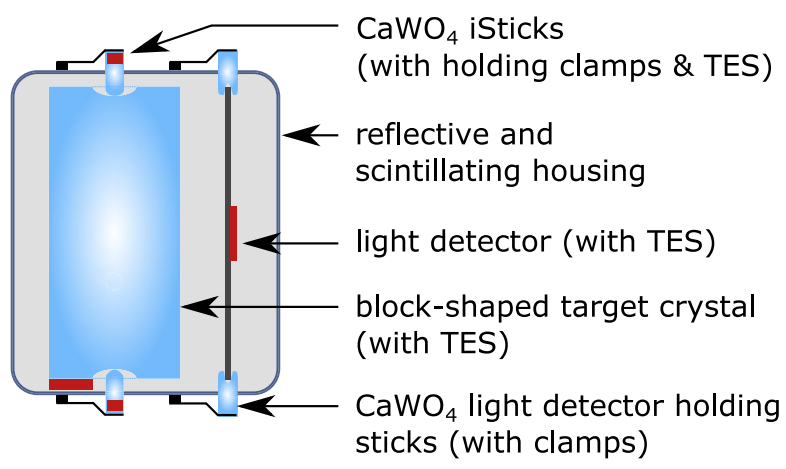

FIG. 1. Schematic of a CRESST-III detector module (not to scale). Parts in blue are made of $\mathrm{CaWO}_{4}$; the TESs are sketched in red. The block-shaped target (absorber) crystal has a mass of $\sim 24 \mathrm{~g}$; its dimensions are $(20 \times 20 \times 10) \mathrm{mm}^{3}$. It is held by three instrumented $\mathrm{CaWO}_{4}$ holding sticks (iSticks), two at the bottom and one on top. Three noninstrumented $\mathrm{CaWO}_{4}$ holding sticks keep the square-shaped silicon-on-sapphire light detector in place. Its dimensions are $(20 \times 20 \times 0.4) \mathrm{mm}^{3}$.

\section{B. CRESST-III detector design}

The $\mathrm{CaWO}_{4}$ crystal of a CRESST-III detector module has a size of $(20 \times 20 \times 10) \mathrm{mm}^{3}$ and a mass of $\sim 24 \mathrm{~g}$ (23.6 $\mathrm{g}$ for detector A). A schematic drawing is shown in Fig. 1. The target crystal is held by three $\mathrm{CaWO}_{4}$ sticks, each with a length of $12 \mathrm{~mm}$, a diameter of $2.5 \mathrm{~mm}$, and a rounded tip of approximately $2-3 \mathrm{~mm}$ in radius. The sticks are themselves instrumented with a TES, thus denoted iSticks. This novel, instrumented detector holder allows an identification and veto of interactions taking place in the sticks which might potentially cause a signal in the target crystal due to phonons propagating from the stick to the main absorber through their contact area. Since we veto interactions in any of the sticks, the three iSticks are connected in parallel to one SQUID, thus substantially reducing the number of necessary readout channels [8].

Each target crystal is paired with a cryogenic light detector, matched to the size of the target crystal, consisting of a $0.4 \mathrm{~mm}$ thick square silicon-on-sapphire wafer of $20 \mathrm{~mm}$ edge length, also held by $\mathrm{CaWO}_{4}$ sticks and equipped with a TES. However, an instrumentation of these sticks is not needed as events within them would cause quasi-light-only events which are outside the acceptance region for the dark matter search (see Sec. III D). ${ }^{1}$

The remaining ingredient to achieve a fully active surrounding of the target crystal is the reflective and scintillating Vikuiti foil encapsulating the ensemble of target crystal and light detector. Such a fully active design ensures that surface-related backgrounds, in particular, surface $\alpha$-decays, are identified and subsequently excluded

\footnotetext{
${ }^{1} \mathrm{~A}$ small fraction of the light emitted by the stick might be absorbed by the target crystal, creating a small phonon signal therein; thus these events are denoted quasi-light-only.
} 
from the dark matter analysis. A detailed study of the event classes arising from the iSticks and the light detector holding sticks is beyond the scope of this work; performance studies on the parallel TES readout may be found in [9].

Each TES is paired with an ohmic heater. Through the injection of large pulses to this heater (control pulses) which heat the TES completely out of its transition, the current operating point in the transition curve is evaluated for each detector. The measured height of the control pulses is fed to an online feedback loop which maintains a constant operating point by adjusting the heating power accordingly.

In addition, small heater pulses of different sizes (test pulses) are injected periodically to precisely determine the detector response curve over the full dynamic range including potential, small, time variations. The heaters are calibrated in terms of equivalent deposited energy by comparison of the detector response to $\gamma$-lines (see Sec. III A).

\section{Dead-time free recording and offline triggering}

The TESs are read out with SQUIDs whose signals are first passed through amplifiers close to the cryostat and then fed through the Faraday cage wall (filtered). In CRESST-III, the existing hardware-triggered data acquisition (DAQ) is extended by transient digitizers, allowing for a dead-time free, continuous recording of the signals with a resolution of 16 bit for a range of $[-10,10] \mathrm{V}$ and a sampling rate of $25 \mathrm{kHz}$. Recording the full signal stream allows the use of an offline software trigger adapted to each detector. Our software trigger is based on the optimum filter or Gatti-Manfredi filter [10] successfully used e.g., by the CUORE experiment $[11,12]$. The optimum filter maximizes the signal-to-noise ratio by comparing the frequency power spectrum of noise samples to that of an averaged pulse (a standard event). More weight is then given to pulselike frequencies compared to those dominantly appearing in the noise samples. More details on the continuous DAQ and the signal processing with the optimum filter may be found in [13].

The complete stream is filtered with the optimum filter, and a trigger is fired whenever the filter output for the phonon or light channel exceeds a certain threshold value. For each channel we select a record window of $655.36 \mathrm{~ms}$ for further analysis. Phonon pulses have rise times of $1.4 \mathrm{~ms}$ and decay times of $15 \mathrm{~ms}$ and $\sim 100 \mathrm{~ms}$ for the fast and slow components, respectively. Light pulses rise about 2 times faster with similar decay times.

More details may be found in [13]. The output of the optimum filter is not only used for the software triggering, but it is also the basis of the energy reconstruction (see Sec. III), yielding a precise value of the threshold in energy units.

\section{Optimal trigger threshold}

Thanks to the continuously recorded data stream, the trigger threshold can be optimized based on a predefined

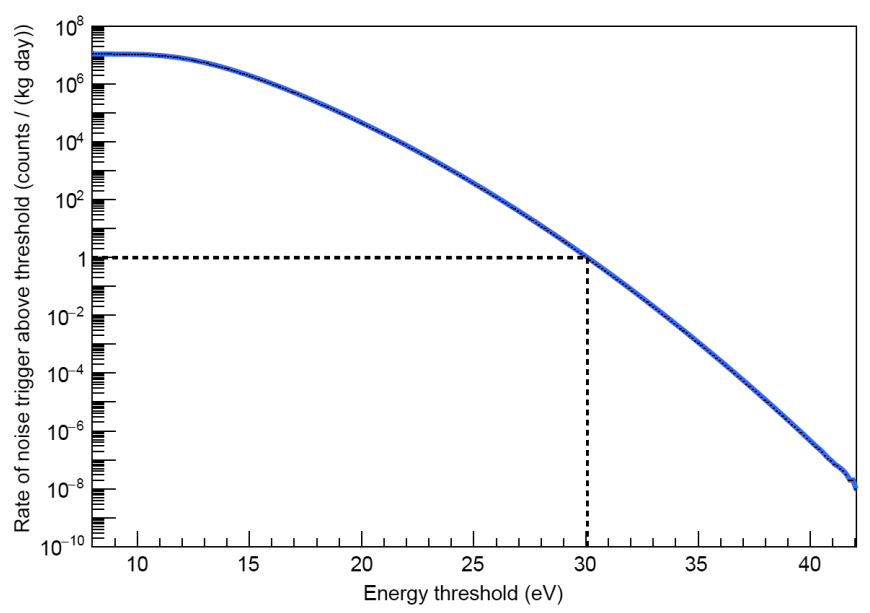

FIG. 2. Number of expected noise triggers surviving event selection per $\mathrm{kg}$ day as a function of a chosen trigger threshold for detector A. The threshold chosen for this work is indicated by the dashed line at $30.1 \mathrm{eV}$.

criterion as described in [14]. For this analysis one noise event surviving event selection per $\mathrm{kg}$ day was allowed, corresponding to an average trigger threshold of $30.1 \mathrm{eV}$ (see Fig. 2). The trigger threshold is fixed in terms of optimum filter output voltage. Its conversion to energy may vary due to small time variations of the detector response. This effect was fully accounted for in the simulation of the expected dark matter spectrum (see Sec. III E), based on the time-dependent detector response measured with the test pulses.

\section{ENERGY CALIBRATION AND EVENT SELECTION}

\section{A. Energy calibration}

For CRESST-III, challenges arise from the greatly enhanced sensitivity. This leads to strong saturation effects at the $122 \mathrm{keV} \gamma$-line from an external ${ }^{57} \mathrm{Co}$ source used in former CRESST phases to calibrate the detectors. The saturation is caused by the TES reaching its normalconducting state above which a further temperature increase will not lead to larger pulse heights [15]. To directly probe the linear, nonsaturated range of the detectors, lower $\gamma$-ray energies would be required. Those, however, cannot efficiently penetrate the cryostat. Therefore, we perform an initial, approximate calibration using the $\mathrm{K}_{\alpha 1}$ and $\mathrm{K}_{\alpha 2}$ escape peaks of tungsten with a weighted mean energy of $63.2 \mathrm{keV}$, and later we fine-adjust by scaling to the $11.27 \mathrm{keV}$ peak (Hf $\mathrm{L}_{1}$ shell, [16]). The latter originates from cosmogenic activation of tungsten and is visible in all CRESST-III detectors (see Sec. III).

The optimum filter offers a better resolution for the energy reconstruction than the standard event fit [13], as used in previous analyses. With the optimum filter we achieve a baseline resolution, i.e., resolution at zero energy, 
of $\sigma=4.6 \mathrm{eV}$. However, saturation effects, which cannot be compensated by the optimum filter algorithm, set in at $2.5 \mathrm{keV}$ with complete signal saturation around $75 \mathrm{keV}$. To partially overcome this limitation, we compare the amplitude determined by the optimum filter to the amplitude determined by a truncated standard event fit. The relation between these two quantities is obtained from high statistics neutron calibration data and allows us to extend the usable range for the optimum filter up to $16 \mathrm{keV}$. Above $16 \mathrm{keV}$, the saturation is too large to be reasonably corrected by this procedure, and we therefore restrict our dark matter analysis to energies below $16 \mathrm{keV}$.

\section{B. Light yield description using neutron calibration data}

To discriminate different types of particle interactions, we define the light yield of an event as the ratio of the energies deposited in the light and phonon channel: $L Y=E_{l} / E_{p}$.

For this analysis, the phonon energy $E_{p}$ is considered to be the total deposited energy of an event; this approximation neglects any small possible dependence of $E_{p}$ on the event type and is motivated in Appendix VII D.

Figure 3 shows the events surviving the selection criteria (see Sec. III D) in the AmBe neutron calibration data. The solid blue lines mark the $90 \%$ upper and lower boundaries of the $\beta / \gamma$-band. The red and green lines mark the bands expected for recoils off oxygen and tungsten, respectively. The calcium band lies in between the oxygen and the tungsten band and is not drawn for clarity.

The description of the bands is done according to [17]. The mean of the Gaussian $\beta / \gamma$-band is given by a linear function plus a term accounting for the nonproportionality

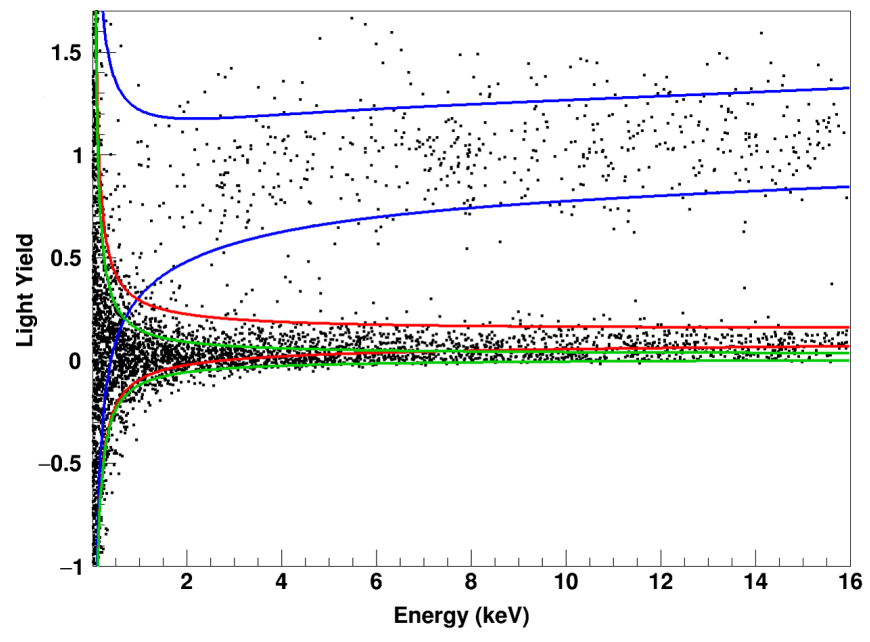

FIG. 3. Neutron calibration data for detector A in the light yield versus energy plane. We fit these data to determine the bands for $\beta / \gamma$-events (blue), nuclear recoils off oxygen (red), and tungsten (green), where the respective lines correspond to the upper and lower $90 \%$ boundaries of the respective band. The band description follows [17]. effect causing the bending down of the $\beta / \gamma$-band towards low energies [18]. Quenching factors quantify the reduction in light output of a certain event type compared to a $\beta / \gamma$ event of the same deposited energy. They were precisely measured in [17] and are used to calculate the nuclear recoil bands.

For the present work we fit the neutron calibration data ( $\beta / \gamma$-band plus nuclear recoil bands) utilizing an unbinned maximum likelihood approach. Using the neutron calibration data, instead of the dark matter data directly, has several advantages. First, it was found in [17] that different crystals exhibit a slightly different quenching for nuclear recoils which, however, commonly affects all three nuclear recoil bands. In previous analyses we determined this common shift separately from the likelihood fit by looking at oxygen scatters in neutron calibration data with energies above $150 \mathrm{keV}$. The new likelihood fit, instead, directly obtains the common shift from the position of the nuclear recoil bands. The second advantage of performing the fit on neutron calibration data is a more densely populated $\beta / \gamma$ band compared to dark matter data (compare Figs. 3 and 5).

We note that the term $\beta / \gamma$-band should more correctly be denoted $\beta$-band, as $\gamma$ rays are known to produce slightly less scintillation light than $\beta$ particles [18]. This is particularly apparent for the discrete $\gamma$ populations at $2.6 \mathrm{keV}$ and $11.27 \mathrm{keV}$ in Fig. 5 that are clearly centered below the $\beta / \gamma$-band. We model this effect in the new maximum likelihood fit. However, for means of clarity and convention, we stick to the term $\beta / \gamma$-band.

The neutron calibration data also confirm that nuclear recoils and $\beta / \gamma$-events have a negligible pulse-shape difference. This justifies the use of a single standard event as the basis for triggering and energy calibration.

\section{Data preselection}

With stops in data taking for refills of cryogenic liquids, there are three data segments per week in standard data taking mode. In the rare cases of synchronization issues between the two data acquisitions (hardware-triggered and continuous), the affected segments are discarded. This includes the segment containing the event at $\sim 3.7 \mathrm{keV}$ seen in the region of interest in the analysis of [5]. To establish the analysis procedure we define a nonblind training set by random selection of $20 \%$ of the collected data segments. The procedure is then blindly applied to the remaining $80 \%$. All results presented in this work, apart from the calibration steps, refer to the latter, "blind" dark matter data set.

Part of the data had already been unblinded in [5] but only for energies above $100 \mathrm{eV}$. For the analysis of the continuous data presented in this work, some data selection criteria had to be slightly adjusted in value to account for differences in the data processing.

A binned rate cut is applied to remove periods of abnormally high rate, mainly due to small electronic 
disturbances, which were found to cluster in time. In total, this rate cut excludes $14 \%$ of measuring time.

We remove time intervals where the measured control pulse height deviates by more than $3 \sigma$ from the mean of its Gaussian-shaped distribution. The additional amount of measuring time removed by this stability cut is $3 \%$.

\section{Event selection}

Events where only the light channel triggered are removed from the dark matter analysis. The veto information from the instrumented $\mathrm{CaWO}_{4}$ sticks is exploited by removing all events with a pulse height in the iStick channel above noise. Additionally, we apply dedicated cuts to remove artifacts mimicking a pulse and events with distorted baselines which would potentially impair the energy reconstruction.

We quantify deviations of a real pulse from its nominal pulse shape during the application of the optimum filter algorithm. This is done by calculating the RMS difference between the filtered real pulse and the filtered standard event, where the latter is scaled to the amplitude of the real pulse. To increase the sensitivity to such deviations, we restrict this calculation to a window of $\pm 30 \mathrm{~ms}$ around the peak of the pulse. We call this quantity $\mathrm{RMS}_{\mathrm{OF}}$.

The RMS of the truncated standard event fit $\left(\mathrm{RMS}_{\mathrm{SEF}}\right)$ describes the agreement between a measured event and the standard event in the linear part of the detector response. This fit is performed simultaneously for the phonon and light channel.

$\mathrm{RMS}_{\mathrm{OF}}$ was found to be less affected by changing noise conditions compared to $\mathrm{RMS}_{\mathrm{SEF}}$, which indicates that $\mathrm{RMS}_{\mathrm{OF}}$ is more sensitive to the real pulse shape than $\mathrm{RMS}_{\mathrm{SEF}}$. However, for energies above $2.5 \mathrm{keV}$ (see Sec. III A) saturation effects start to deteriorate $\mathrm{RMS}_{\mathrm{OF}}$ while $\mathrm{RMS}_{\mathrm{SEF}}$ remains unaffected. For this reason we apply cuts on both RMS quantities, aiming to remove events deviating from the nominal pulse shape.

A conservative muon veto cut is applied by rejecting detector events in a time interval of $[-5 \mathrm{~ms},+10 \mathrm{~ms}]$ around a muon veto trigger. Most of the events triggering the muon veto are not muons but are due to radioactivity in the muon veto panels or the PMTs which does not penetrate the shielding. Thus, this cut almost exclusively removes randomly coincident events. The total loss is $7.6 \%$ of measurement time, which is consistent with the muon veto trigger rate of $5.2 \mathrm{~Hz}$. The cut values are conservatively based on considerations of detector time resolutions; no apparent correlation between muon panel hits and detector events was found. An additional cut on coincidences between detector $\mathrm{A}$ and other detectors, using a coincidence window of $\pm 10 \mathrm{~ms}$, is applied, which causes negligible overall dead time and removes no further events in the acceptance region (see Sec. IV). The main purpose of this cut is to remove potential neutron events which have a certain probability to cause energy deposits in multiple detectors, in contrast to dark matter particles.
The total exposure of the dark matter data set after cuts amounts to $3.64 \mathrm{~kg}$ days; the average survival probability for signal events, neglecting energy dependence, lies at approximately $65 \%$ (see next section).

\section{E. Efficiency or signal survival probability}

To determine the probability for a valid signal to be triggered and survive the selection criteria, we pass simulated signal events through the complete analysis chain. The simulated events are created by superimposing the standard event onto the continuous data stream at randomly selected points in time. The events are simulated with high statistics $\left(\sim 6.5 \times 10^{6}\right.$ events for the full dark matter data set) and scaled in height corresponding to a flat energy spectrum from 0 to $20 \mathrm{keV}$.

As the timing of the simulated pulses is random and they are processed in the analysis chain exactly the same way as real pulses, the resulting loss in the simulated spectrum accounts for all artifacts on the stream, as well as cut effects, pileup between events, and dead time due to injected heater pulses, providing an elegant and straightforward way of determining the signal survival probability. In addition, this procedure implicitly accounts for potential time dependencies such as changing noise conditions. It should be noted, however, that pulse saturation effects are not taken into account in the simulation. This implies that the optimum filter amplitude for simulated pulses behaves strictly linearly, and a linearization using truncated fit results as discussed in Sec. III A is not performed.

Figure 4 shows the efficiency in bins of $1 \mathrm{eV}$ where the efficiency is defined as the ratio of surviving events to simulated events in the respective bin. The spectrum in light gray corresponds to all triggered events, the one in dark gray to events remaining after applying all selection criteria. As a cross-check we model the threshold with an error function depicted in red. Its fit yields a value for the threshold of $(30.0 \pm 0.1) \mathrm{eV}$ with a width of $\sigma=(5.3 \pm 0.2) \mathrm{eV}$; both values agree with expectations for the optimum trigger (see Sec. II D) within uncertainties. Two features become apparent for the light gray trigger efficiency. First, there is a pedestal of $12 \%$ originating from pileup of simulated events with previous large energy deposits or injected heater calibration pulses. Such events are efficiently rejected by our selection cuts; thus the pedestal vanishes for the efficiency after cuts. Second, pulses corresponding to energy deposits of less than $\sim 0.4 \mathrm{keV}$ have a higher probability to be hidden by filter effects from a close-by optimal-filtered control pulse. ${ }^{2}$

\footnotetext{
${ }^{2}$ Typically, the optimum filter shows one global maximum at the position of the pulse and several local maxima before and after the main pulse $[11,13]$. The size of these local maxima for a control pulse (maximal possible pulse height) approximately equals the size of a $0.4 \mathrm{keV}$ energy deposition.
} 


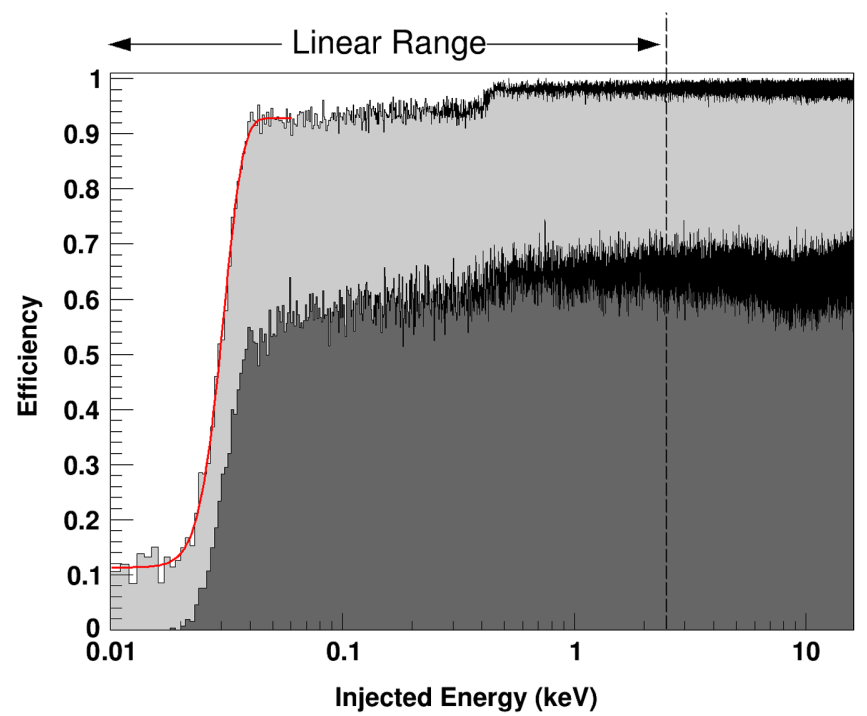

FIG. 4. Efficiency obtained from simulated events and defined as the probability for a valid signal event to be triggered (light gray) and pass the selection criteria (dark gray) as a function of injected (simulated) energy. The red line is a fit of the threshold with an error function, confirming the claimed value of $30.1 \mathrm{eV}$. The vertical dashed line indicates the limit of linear detector response at $2.5 \mathrm{keV}$.

In order to obtain a dark matter exclusion limit, we need to know what the expected dark matter signal looks like after triggering, energy reconstruction, and event selection. We simulate this by injecting artificial pulses into the continuous stream that follow the pulse height distribution of the expected recoil spectrum for each dark matter particle mass (the dark matter model will be discussed in Sec. V). This method automatically includes all relevant aspects, in particular, triggering efficiency and energy resolution, thus resulting in a dark matter recoil spectrum as it would be seen by our detector. It should be explicitly noted that this newly implemented method overcomes the necessity of an analytic modeling of the detector response, in particular, of the finite energy resolution. This represents a simplification in the extraction of dark matter results from the data, but above all, it avoids uncertainties introduced by the model of the detector response and/or the determination of the efficiencies of the analysis pipeline.

To save computation time we perform only one simulation, with a uniform energy distribution from $0 \mathrm{keV}-$ $20 \mathrm{keV}$, and re-weigh each simulated event according to the expected recoil spectrum for a specific dark matter particle mass. We reject simulated events whose reconstructed energies differ by more than 2 standard deviations from the injected or simulated energies. This criterion defends against the impact of single outliers caused by pileup of a simulated event with a real particle event. Such a pileup may result in an overestimate of the survival probability of very small energy deposits.

\section{DARK MATTER DATA SET}

The data used for dark matter analysis were taken between October 2016 and January 2018. The gross exposure before cuts is $5.6 \mathrm{~kg}$ days. We ensure the robustness of our dark matter results by not making use of subthreshold energies, i.e., energies below $30.1 \mathrm{eV}$, where the trigger efficiency is $50 \%$.

\section{A. Light yield}

Figure 5 shows the dark matter data in the light yield versus energy plane after application of all the cuts described before. In accordance with Fig. 3, the blue, red, and green bands correspond to $\beta / \gamma$-events and nuclear recoils off oxygen and tungsten, respectively. The red dashed line depicts the mean of the oxygen band, which also marks the upper boundary of the acceptance region, shaded in yellow. The lower bound of the acceptance region is the $99.5 \%$ lower boundary of the tungsten band; its energy span is from the threshold of $30.1 \mathrm{eV}$ to $16.0 \mathrm{keV}$. Events in the acceptance region (highlighted in red) are treated as potential dark matter candidate events. We restrict the energy range to $16 \mathrm{keV}$ for this analysis since for higher energies the energy reconstruction cannot be based on the optimum filter method due to saturation effects. This choice, however, hardly affects the sensitivity for the low dark matter particle masses of interest. The choice for the acceptance region was fixed a priori before

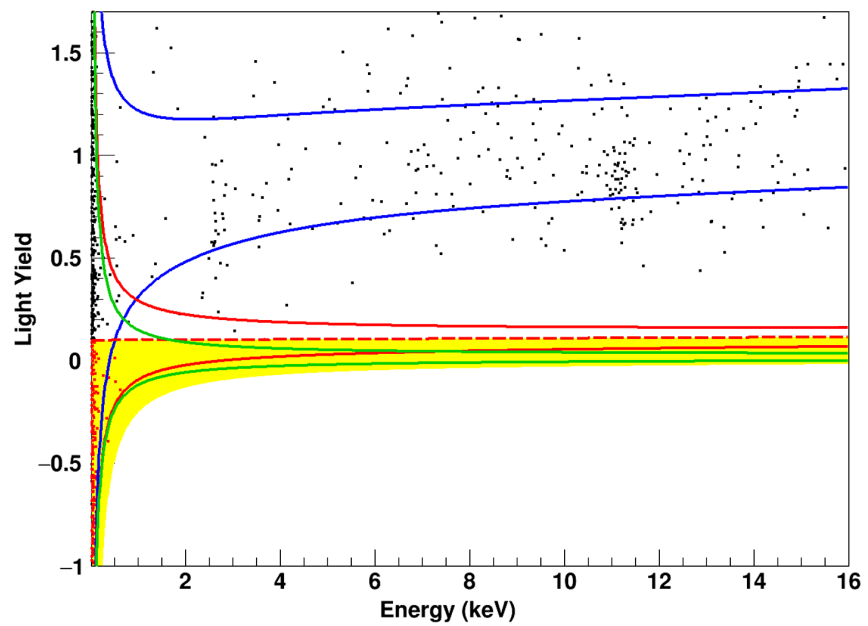

FIG. 5. Light yield versus energy of events in the dark matter data set, after selection criteria are applied (see Sec. III D). The blue band indicates the $90 \%$ upper and lower boundaries of the $\beta / \gamma$-band; red and green show the same for oxygen and tungsten, respectively. The yellow area denotes the acceptance region reaching from the mean of the oxygen band (red dashed line) down to the $99.5 \%$ lower boundary of the tungsten band. Events in the acceptance region are highlighted in red. The position of the bands is extracted from the neutron calibration data as shown in Fig. 3. A zoom to the low-energy region is given in Appendix A 2. 
unblinding the data. We do not include the full oxygen recoil band in the acceptance region because the gain in expected signal is too small to compensate for the increased background leakage from the $\beta / \gamma$-band.

\section{B. Energy spectrum}

The corresponding energy spectrum is shown in Fig. 6 with events in the acceptance region highlighted in red. In both Figs. 5 and 6, event populations at $2.6 \mathrm{keV}$ and $\sim 11 \mathrm{keV}$ are visible. These originate from cosmogenic activation of the detector material and subsequent electron capture decays:

$$
{ }^{182} \mathrm{~W}+\mathrm{p} \rightarrow{ }^{179} \mathrm{Ta}+\alpha, \quad{ }^{179} \mathrm{Ta} \stackrel{\mathrm{EC}}{\longrightarrow}{ }^{179} \mathrm{Hf}+\gamma .
$$

The latter decay has a half-life of 665 days, which implies a decreasing rate over the course of the measurement after initial exposure of the detector material. The energies of the lines correspond to the $\mathrm{L}_{1}$ and $\mathrm{M}_{1}$ shell binding energies of ${ }^{179} \mathrm{Hf}$ with literature values of $\mathrm{E}_{\mathrm{M}_{1}}=$ $2.60 \mathrm{keV}$ and $\mathrm{E}_{\mathrm{L}_{1}}=11.27 \mathrm{keV}$, respectively [19]. An $\mathrm{M}_{1} / \mathrm{L}_{1}$ ratio of $\sim 0.285$ was measured, which is in good agreement with the literature value of 0.281 [19]. As already mentioned in Sec. III A, the clearly identifiable $11.27 \mathrm{keV}$ line was used to fine-adjust the energy scale and therefore to give accurate energy information in the relevant low-energy regime. These features were already observed in CRESST-II [16,20]. Additionally, a population of events at $\sim 540 \mathrm{eV}$ is visible, which hints at electron capture (EC) decays from the $\mathrm{N}_{1}$ shell of ${ }^{179} \mathrm{Hf}$ with a literature value of $\mathrm{E}_{\mathrm{N}_{1}}=538 \mathrm{eV}$ [19]. However, the expected $\mathrm{N}_{1} / \mathrm{M}_{1}$ intensity ratio of 0.27 [19] suggests that only $2-3$ events can be explained by this decay. The energy is also compatible with

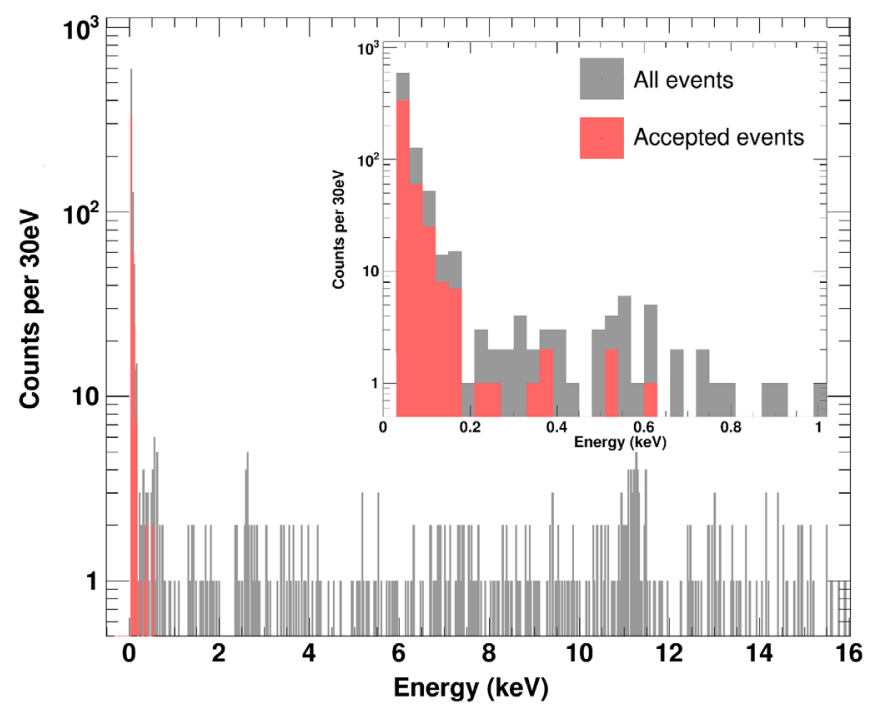

FIG. 6. Energy spectrum of the dark matter data set with lines visible at $2.6 \mathrm{keV}$ and $11.27 \mathrm{keV}$ originating from cosmogenic activation of ${ }^{182} \mathrm{~W}$ [16]. Gray is for all events; red is for events in the acceptance region (see Fig. 5).
$\mathrm{K}_{\alpha, 1}$ and $\mathrm{K}_{\alpha, 2}$ lines of oxygen $(\sim 525 \mathrm{eV})$, which is a main component of the detector material and also abundant in the reflective foil.

The background rate in the energy range from 1 to $16 \mathrm{keV}$ is 5.1 counts $/(\mathrm{keV} \mathrm{kg}$ day) subtracting the aforementioned gamma lines and is 6.63 counts / ( $\mathrm{keV} \mathrm{kg} \mathrm{day)} \mathrm{when} \mathrm{includ-}$ ing them. This value is almost 2 times higher than the best achieved background level of 3.51 counts/( $\mathrm{keV} \mathrm{kg} \mathrm{day)}$ (for [1-40] keV) [16]. While this discrepancy is not fully understood, a recent Monte Carlo simulation of the $\beta / \gamma$ background in CRESST [21] indicates that a substantial fraction of the remaining background has its origin in the $\mathrm{CaWO}_{4}$ crystal itself.

Following the analysis of [4] we expect a neutron background of $\ll 1$ count for the given detector; however, a more credible figure may only be provided by a currently ongoing neutron Monte Carlo simulation. For the given detector, all events in the acceptance region that are flagged by the iStick veto are also removed by the quality and RMS cuts on the phonon and light channel due to their different pulse shape. The stick-holding scheme has proven to be extremely effective, vetoing any surface-related events (see [22]); thus we neither expect degraded alphas nor $\mathrm{Pb}$-recoil events in the analyzed energy range.

Below $200 \mathrm{eV}$, an excess of events above the flat background is visible, which appears to be exponential in shape. Due to decreasing discrimination at low energies, it cannot be determined whether this rise is caused by nuclear recoils or $\beta / \gamma$ events (see Figs. 5 and 6). It should be emphasized that noise triggers are not an explanation for this excess, as it extends too far above the threshold of $30.1 \mathrm{eV}$. According to the definition of the trigger condition in Sec. II D, the expected number of noise triggers for the full data set would be around 3.6. We observe an excess of events at lowest energies in all CRESST-III detector modules with thresholds below $100 \mathrm{eV}$; the shape of this excess varies for different modules, which argues against a single common origin of this effect. No clustering of events in time from the excess populations is observed.

\section{RESULTS}

We use the Yellin optimum interval algorithm $[23,24]$ to extract an upper limit on the dark matter-nucleus scattering cross section. In accordance with this method, we consider all 441 events inside the acceptance region to be potential dark matter interactions; no background subtraction is performed.

The anticipated dark matter spectrum follows the standard halo model [25] with a local dark matter density of $\rho_{\text {DM }}=0.3\left(\mathrm{GeV} / \mathrm{c}^{2}\right) / \mathrm{cm}^{3}$, an asymptotic velocity of $v_{\odot}=$ $220 \mathrm{~km} / \mathrm{s}$, and an escape velocity of $v_{\text {esc }}=544 \mathrm{~km} / \mathrm{s}$. Form factors, which are hardly relevant given the low transferred momenta here, follow the model of Helm [26] in the parametrization of Lewin and Smith [27].

The result of the present analysis on elastic scattering of dark matter particles off nuclei is depicted in solid red in 


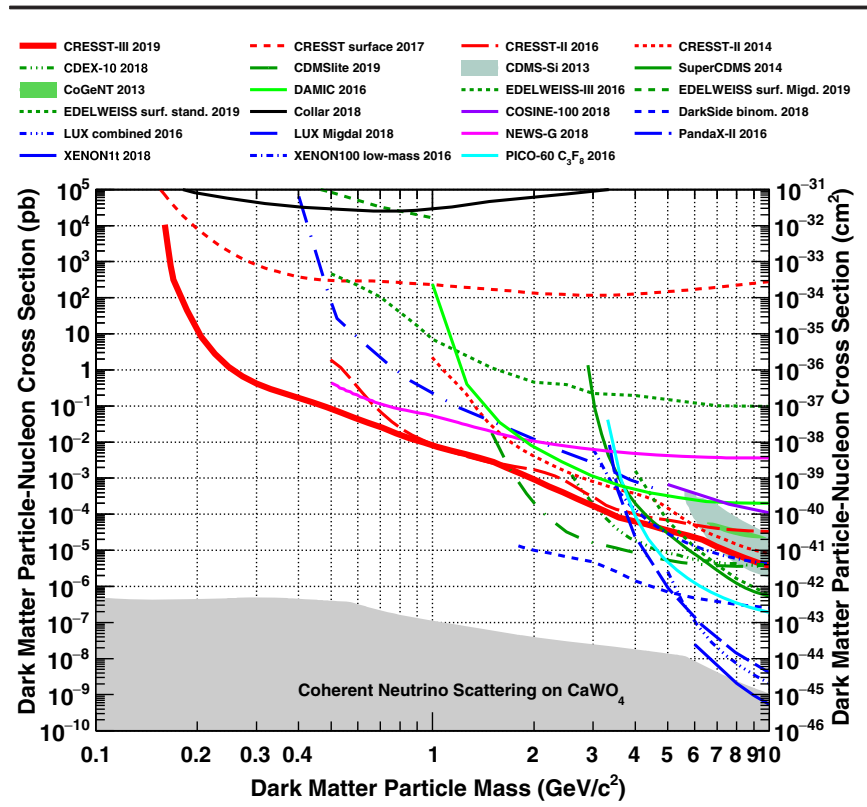

FIG. 7. Experimental results on elastic, spin-independent, dark matter-nucleus scattering depicted in the cross section versus dark matter particle mass plane. If not specified explicitly, results are reported with $90 \%$ confidence level (C.L.). The result of this work is depicted in solid red with the most stringent limit between masses of (0.16-1.8) GeV/c $\mathrm{c}^{2}$. The previous CRESST-II result is depicted in dashed red [29]; the red dotted line corresponds to a surface measurement performed with a gram-scale $\mathrm{Al}_{2} \mathrm{O}_{3}$ detector [28]. We use color coding to group the experimental results: green for exclusion limits (CDEX-10 [30], CDMSlite [31], DAMIC [32], EDELWEISS [33,34], SuperCDMS [35]) and positive evidence [CDMS-Si (90\% C.L.) [35], CoGeNT (99\% C.L.) [36]) obtained with solid state detectors based on silicon or germanium, blue for liquid noble gas experiments based on argon or xenon (DarkSide [37], LUX [38,39], Panda-X [40], Xenon100 [41], Xenon1t [42]), violet for COSINE-100 (NaI) [43], black for Collar $(\mathrm{H})$ [44], magenta for the gaseous spherical proportional counter NEWS-G $\left(\mathrm{Ne}+\mathrm{CH}_{4}\right)$ [45], and cyan for the super-heated bubble chamber experiment PICO $\left(\mathrm{C}_{3} \mathrm{~F}_{8}\right)$ [46]. The gray region marks the so-called neutrino floor calculated for $\mathrm{CaWO}_{4}$ in [47].

Fig. 7 in comparison to the previous CRESST-II exclusion limit in dashed red and results from other experiments (see caption and legend of Fig. 7 for details). The red dotted line corresponds to a surface measurement with a $0.5 \mathrm{~g} \mathrm{Al}_{2} \mathrm{O}_{3}$ crystal achieving a threshold of $19.7 \mathrm{eV}$ using CRESST technology [28].

The sensitivity of the analyzed detector module $A$ is limited by the observed exponential background for masses lower than $7 \mathrm{GeV} / \mathrm{c}^{2}$. The other detector modules either show a similar background or do not reach the required thresholds to unambiguously observe it, leading to less stringent limits.

The improvement in the achieved nuclear recoil threshold, in the respectively best performing detectors, from $0.3 \mathrm{keV}$ for CRESST-II to $30.1 \mathrm{eV}$ for CRESST-III, yields a factor of more than 3 in terms of reach for low masses, down to $0.16 \mathrm{GeV} / \mathrm{c}^{2}$. At $0.5 \mathrm{GeV} / \mathrm{c}^{2}$ we improve existing limits by a factor of 6 (30) compared to NEWS-G (CRESST-II). In the range $(0.5-1.8) \mathrm{GeV} / \mathrm{c}^{2}$ we match or exceed the previously leading limit from CRESST-II.

\section{CONCLUSION}

In this article, we report newly implemented data processing methods, featuring, in particular, the optimum filter technique for software triggering and energy reconstruction. This allows one to make full use of the data down to threshold. The best detector operated in the first run of CRESST-III (05/2016-02/2018) achieves a threshold as low as $30.1 \mathrm{eV}$ and was, therefore, chosen for the analysis presented.

In comparison to previous CRESST measurements, an indication of a $\gamma$-line at approximately $540 \mathrm{eV}$ could be observed. The reappearance of known lines corroborates the analysis of background components outlined in [16], as well as the energy calibration in this work.

At energies below $200 \mathrm{eV}$ we observe a rising event rate which is incompatible with a flat background assumption and seems to point to a so-far unknown contribution. Recently, dedicated hardware tests with upgraded detector modules were underway to illuminate its origin.

We present exclusion limits on elastic dark matter particle-nucleus scattering, probing dark matter particle masses below $0.5 \mathrm{GeV} / \mathrm{c}^{2}$ and down to $0.16 \mathrm{GeV} / \mathrm{c}^{2}$.

\section{ACKNOWLEDGMENTS}

We are grateful to LNGS for their generous support of CRESST. We would like to thank Timon Emken and Riccardo Catena for the constructive discussion and their valuable input on the effect of Earth shielding. This work has been supported through the DFG by the SFB1258 and the Excellence Cluster Universe, and by the BMBF: 05A17WO4 and 05A17VTA.

\section{APPENDIX A: SPIN-DEPENDENT SCATTERING, DATA QUALITY CHECKS, SYSTEMATIC UNCERTAINTIES}

\section{Results on spin-dependent interactions}

In this article we present first results of CRESST-III on spin-independent elastic dark matter-nucleus scattering. However, it deserves to be noted that the isotope ${ }^{17} \mathrm{O}$ yields sensitivity for spin-dependent neutron-only interactions. The theoretical framework, as well as the calculation of the expected rate, exactly follows [48]; thus, just the result is given here. Compared to [48] the nuclear spin value $(J=+5 / 2)$, the atomic mass number $(A=17)$, and the spin matrix element $\left(\left\langle S_{n}\right\rangle=0.5\right)[49,50]$ are adjusted. We assume the ${ }^{17} \mathrm{O}$ content to follow the minimal natural abundance of $0.0367 \%$ [51] which results in a gross ${ }^{17} \mathrm{O}$ exposure of only $0.46 \mathrm{~g}$ days. Following [52] and 
considering the rock composition of the LNGS overburden [53], we ensured that spin-dependent cross sections of $\mathcal{O}\left(10^{9} \mathrm{pb}\right)$ can be probed over the whole mass range under consideration (red-hatched line). However, a more precise calculation of the upper boundary of the exclusion is left to future work.

It should be stressed that nothing in the analysis chain but the signal expectation was changed when switching from the spin-independent to the spin-dependent case. The result is depicted in Fig. 8 in solid red, together with a result from an above-ground measurement of a $\mathrm{Li}_{2} \mathrm{MoO}_{4}$ crystal in dashed red and exclusion limits from CDMS-lite, LUX, Panda-X, and XENON1t (see caption for references).

Obviously, the small exposure for this measurement, combined with the very low abundance of ${ }^{17} \mathrm{O}$, results in a comparably modest limit for dark matter particles above $1.5 \mathrm{GeV} / \mathrm{c}^{2}$. However, the low nuclear recoil threshold of the presented detector A allows us to explore new parameter space for spin-dependent, neutron-only interactions from dark matter particle masses of $1.5 \mathrm{GeV} / \mathrm{c}^{2}$ down to $0.16 \mathrm{GeV} / \mathrm{c}^{2}$.

\section{Zoomed data plot}

Figure 9 shows a zoom to the low-energy region of Fig. 5.

\section{Rate cut, noise, and disturbances}

Several periods of high rates of electronic disturbances appeared during the run. These periods were removed as

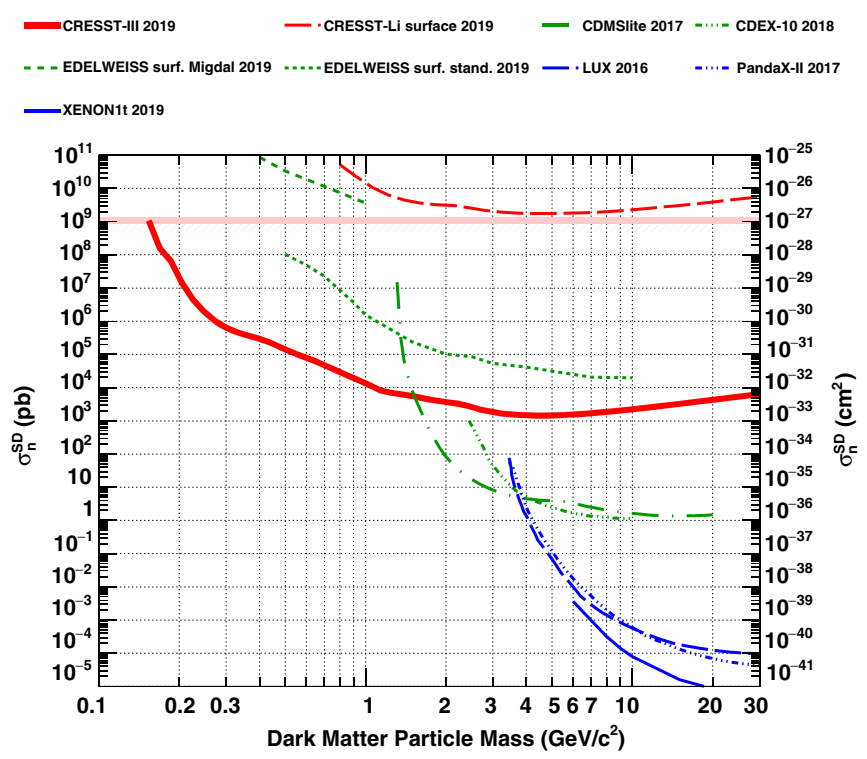

FIG. 8. Results on spin-dependent neutron-only interactions via the isotope ${ }^{17} \mathrm{O}$ in solid red (this work) and a result with ${ }^{7} \mathrm{Li}$ in dashed red [48]. The red hatched line indicates a conservative estimate for the maximal cross section unaffected by effects of Earth shielding. Additionally, we plot results from CDMS-lite and CDEX-10 on ${ }^{73} \mathrm{Ge}$ [30,54], LUX [55], Panda-X [56], and XENON1t [57], all three on ${ }^{129} \mathrm{Xe}$ and ${ }^{131} \mathrm{Xe}$.

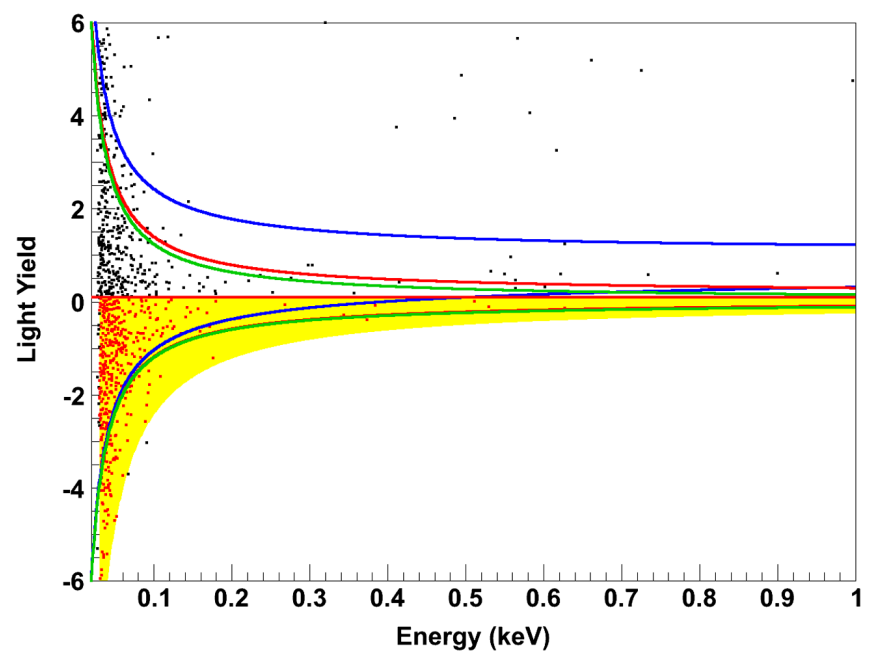

FIG. 9. Zoomed version of Fig. 5 showing the data in the dark matter data set. Yellow shows the acceptance region, blue shows the $\beta / \gamma$-band, and red and green show the oxygen and tungsten recoil bands. For details see Fig. 5 and Sec. III B.

depicted in the Fig. 10, bottom. In some cases, they do, but not necessarily, coincide with higher baseline noise as depicted in Fig. 10, middle and top.

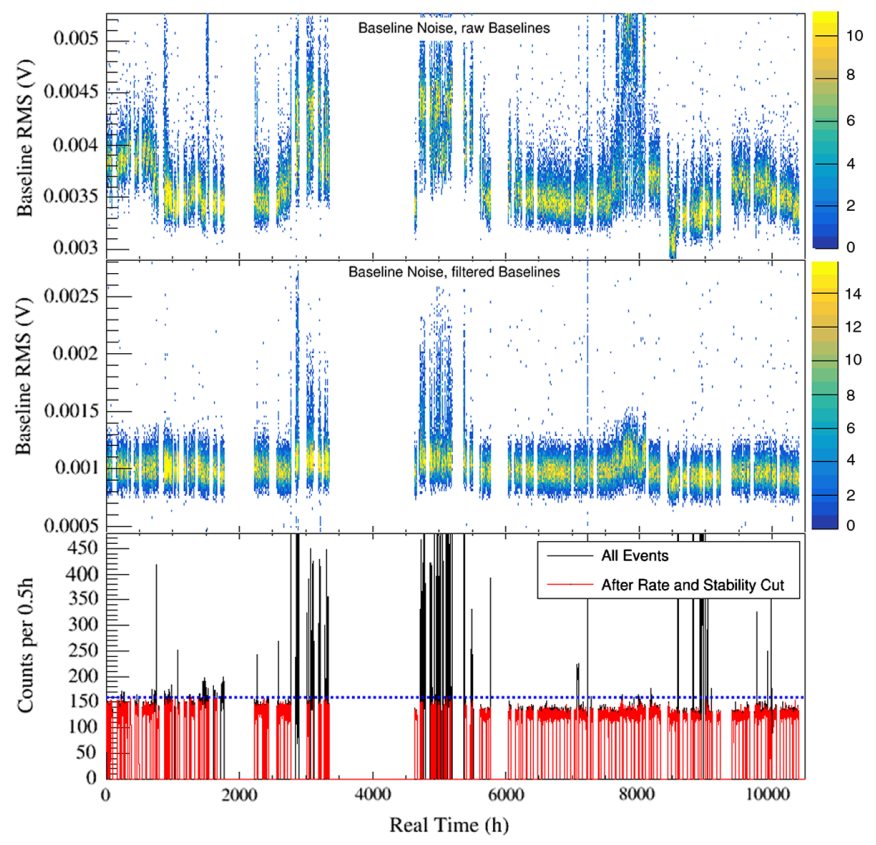

FIG. 10. Time evolution of baseline noise, trigger rate, and rate cut. The baseline noise is calculated from randomly triggered empty baselines. "Empty" baselines that clearly contain part of a pulse are removed. Top: Baseline RMS versus time of the raw recorded baselines. Middle: Baseline RMS versus time after applying the optimum filter to the baseline. Bottom: Rate of triggered events, all events (black), events after rate and stability cut (red); compare Sec. III D. The blue dotted line marks the maximum allowed rate of 160 events $/ 0.5 \mathrm{~h}$. The conversion factor to energy for the RMS is about $4 \mathrm{keV} / \mathrm{V}$. 
The electronic disturbances are excluded as the origin of the high rate of events below $200 \mathrm{eV}$ (see Fig. 6) due to their different pulse shape.

\section{Study of systematic uncertainties}

As discussed in Sec. III the energy scale is adjusted using the $11.27 \mathrm{keV} \gamma$-peak ( $\mathrm{Hf} \mathrm{L}_{1}$ shell). As a consequence, the energy scale is only strictly valid for events with a light yield of 1. In particular, for a nuclear recoil less scintillation light is produced and, thus, more energy remains in the phonon channel leading to an overestimation of the phonon energy. Based on the fact that we measure both energies-phonon $\left(E_{p}\right)$ and light $\left(E_{l}\right)$-one can account for this effect as was shown in [20] by applying the following correction:

$$
E=\eta E_{l}+(1-\eta) E_{p}=[1-\eta(1-L Y)] E_{p} .
$$

In the above equation $\eta$ is the scintillation light efficiency which was determined to be $(6.6 \pm 0.4) \%$ in [20] for a crystal of the same origin as detector A, also grown within the CRESST Collaboration.

However, for very low energies, the baseline noise of the light detector dominates the light signal, preventing a reasonable application of this correction. It should be noted that the resulting overestimation of the nuclear recoil energy scale is conservative as displayed in Fig. 11 where we show the exclusion limit after a reduction of $7 \%$ of all

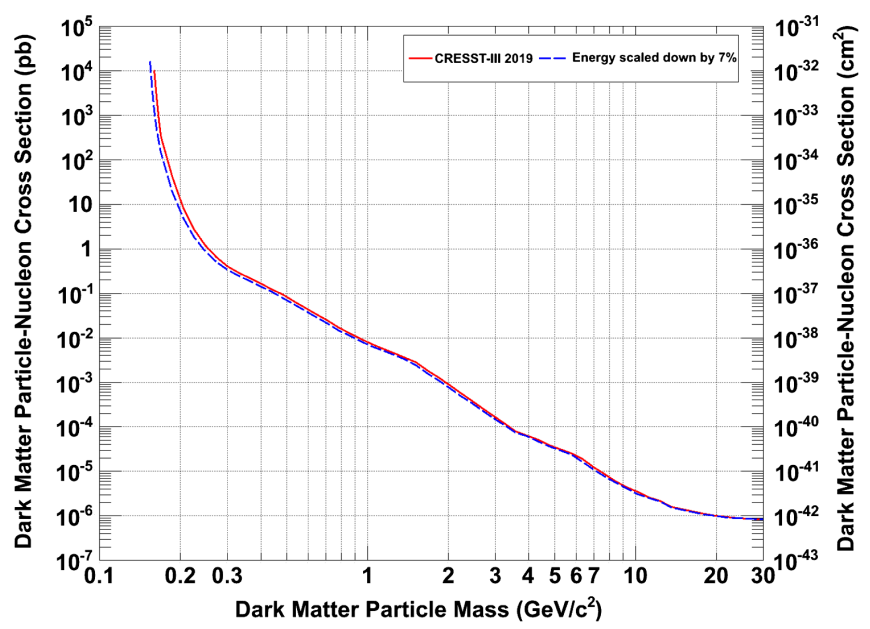

FIG. 11. Illustration of the systematic uncertainty introduced by an overestimation of the nuclear recoil energy scale as a consequence of not correcting for the reduced scintillation light production of nuclear recoils via Eq. (1). We show the result of this work in solid red (also see Fig. 7) and an exclusion limit obtained via scaling of the energy scale by $7 \%$ (dashed blue line), which corresponds to the maximal possible overestimation. It can be seen that this systematic uncertainty has only a minor impact on the exclusion limit. Not applying the correction leads to a conservative exclusion limit for all dark matter particle masses.

event energies. This is the maximal possible systematic uncertainty introduced by not applying the correction outlined in Eq. (1).
[1] M. Tanabashi et al. (Particle Data Group), Phys. Rev. D 98, 030001 (2018).

[2] J. Liu, X. Chen, and X. Ji, Nat. Phys. 13, 212 (2017).

[3] R. Essig, J. Mardon, and T. Volansky, Phys. Rev. D 85, 076007 (2012).

[4] G. Angloher et al., Eur. Phys. J. C 72, 1971 (2012).

[5] F. Petricca et al. (CRESST Collaboration), arXiv:1711 .07692 .

[6] M. Ambrosio et al., Phys. Rev. D 52, 3793 (1995).

[7] G. Angloher, M. Bauer, I. Bavykina, A. Bento, A. Brown, C. Bucci, C. Ciemniak, C. Coppi, G. Deuter, and F. von Feilitzsch, Astropart. Phys. 31, 270 (2009).

[8] R. Strauss et al., Nucl. Instrum. Methods Phys. Res., Sect. A 845, 414 (2017).

[9] J. Rothe, Achieving low thresholds: Cryogenic detectors for low-mass dark matter searches, Master's thesis, Ludwig Maximilian Universität München, 2016.

[10] E. Gatti and P. F. Manfredi, Riv. Nuovo Cimento 9, 1 (1986).

[11] S. D. Domizio, F. Orio, and M. Vignati, J. Instrum. 6, P02007 (2011).

[12] C. Alduino et al., Eur. Phys. J. C 77, 857 (2017).

[13] N. Ferreiro Iachellini, Increasing the sensitivity to low mass dark matter in CRESST-III with a new DAQ and signal processing, Ph.D. thesis, Ludwig Maximilian Universität München, 2019.

[14] M. Mancuso et al., J. Low Temp. Phys. 193, 441 (2018).

[15] G. Angloher et al., Astropart. Phys. 23, 325 (2005).

[16] R. Strauss et al., J. Cosmol. Astropart. Phys. 06 (2015) 030 .

[17] R. Strauss et al., Eur. Phys. J. C 74, 2957 (2014).

[18] R. F. Lang et al., arXiv:0910.4414.

[19] R. Firestone, C. Baglin, and S. Chu, Table of Isotopes: 1999 Update on CD-ROM (Wiley-Interscience, New York, 1999).

[20] G. Angloher et al. (CRESST Collaboration), Eur. Phys. J. C 74, 3184 (2014).

[21] A. H. Abdelhameed et al. (CRESST Collaboration), Eur. Phys. J. C 79, 881 (2019).

[22] R. Strauss et al., Eur. Phys. J. C 75, 352 (2015).

[23] S. Yellin, Phys. Rev. D 66, 032005 (2002).

[24] S. Yellin, Software for computing an upper limit given unknown background (02/2011), http://cdms.stanford.edu/ Upperlimit/.

[25] F. Donato, N. Fornengo, and S. Scopel, Astropart. Phys. 9, 247 (1998).

[26] R. H. Helm, Phys. Rev. 104, 1466 (1956). 
[27] J. D. Lewin and P. F. Smith, Astropart. Phys. 6, 87 (1996).

[28] G. Angloher et al. (CRESST Collaboration), Eur. Phys. J. C 77, 637 (2017).

[29] G. Angloher et al., Eur. Phys. J. C 76, 25 (2016).

[30] H. Jiang et al. (CDEX Collaboration), Phys. Rev. Lett. 120, 241301 (2018).

[31] R. Agnese et al. (SuperCDMS Collaboration), Phys. Rev. D 99, 062001 (2019).

[32] A. Aguilar-Arevalo et al. (DAMIC Collaboration), Phys. Rev. D 94, 082006 (2016).

[33] L. Hehn et al., Eur. Phys. J. C 76, 548 (2016).

[34] E. Armengaud et al. (EDELWEISS Collaboration), Phys. Rev. D 99, 082003 (2019).

[35] R. Agnese et al. (SuperCDMS Collaboration), Phys. Rev. Lett. 112, 241302 (2014).

[36] C. E. Aalseth et al. (CoGeNT Collaboration), Phys. Rev. D 88 (2013).

[37] P. Agnes et al. (DarkSide Collaboration), Phys. Rev. Lett. 121, 081307 (2018).

[38] D. Akerib et al. (LUX Collaboration), Phys. Rev. Lett. 118, 021303 (2017).

[39] D. Akerib et al. (LUX Collaboration), Phys. Rev. Lett. 122, 131301 (2019).

[40] X. Cui et al. (PandaX-II Collaboration), Phys. Rev. Lett. 119, 181302 (2017).

[41] E. Aprile et al. (XENON Collaboration), Phys. Rev. D 94, 092001 (2016).
[42] E. Aprile et al. (XENON Collaboration), Phys. Rev. Lett. 121, 111302 (2018).

[43] G. Adhikari et al. (COSINE-100 Collaboration), Nature (London) 564, 83 (2018).

[44] J. Collar, Phys. Rev. D 98, 023005 (2018).

[45] Q. Arnaud et al., Astropart. Phys. 97, 54 (2018).

[46] C. Amole et al. (PICO Collaboration), Phys. Rev. D 93, 052014 (2016).

[47] A. Gütlein et al., Astropart. Phys. 69, 44 (2015).

[48] A. H. Abdelhameed et al. (CRESST Collaboration), Eur. Phys. J. C 79, 630 (2019).

[49] V. A. Bednyakov and F. Simkovic, Phys. Part. Nucl. 36, 131 (2005).

[50] A. F. Pacheco and D. Strottman, Phys. Rev. D 40, 2131 (1989).

[51] N. E. Holden et al., Pure Appl. Chem. 90, 1833 (2018).

[52] D. Hooper and S. D. McDermott, Phys. Rev. D 97, 115006 (2018).

[53] H. Wulandari, J. Jochum, W. Rau, and F. von Feilitzsch, Astropart. Phys. 22, 313 (2004).

[54] R. Agnese et al. (SuperCDMS Collaboration), Phys. Rev. D 97, 022002 (2018).

[55] D. Akerib et al. (LUX Collaboration), Phys. Rev. Lett. 116, 161302 (2016).

[56] C. Fu et al. (PandaX-II Collaboration), Phys. Rev. Lett. 118, 071301 (2017).

[57] E. Aprile et al. (XENON Collaboration), Phys. Rev. Lett. 122, 141301 (2019). 